\title{
Bayesian change-point estimation in the presence of a single outlier
}

\author{
Cherifa Belkacem and Hocine Fellag \\ Department of Mathematics Faculty of Sciences. University of Tizi Ouzou, Tizi-Ouzou. 15000. \\ Algeria
}

Received 7 December 2011; Accepted 27 March 2012

Copyright (c) 2012, Journal Afrika Statistika. All rights reserved

\begin{abstract}
The Bayesian estimation of the change-point in independent gaussian samples is considered. The impact of an outlier on the performance of the Bayesian procedure is studied and the posterior density under contamination is given. In this paper, we show that, in the case of small samples, an outlier has a significant impact on the estimation of the change-point. However, if the sample size is enough high, the estimation performs well.

Résumé. Dans cet article, nous étudions l'estimation bayesienne d'un point de rupture dans un échantillon gaussien. L'impact d'une contamination sur les performances de la procédure bayesienne est mis en évidence travers l'obtention de la densité a posteriori sous contamination. Nous montrons que cet impact est faible dans le cas asymptotique et n'est significatif que pour des échantillons finis.
\end{abstract}

Key words: Bayesian estimation; Change-point; Gaussian models; Outlier.

AMS 2000 Mathematics Subject Classification : Primary 62F15; Secondary 62F30.

\section{Introduction}

Consider the following model : $X_{1}, X_{2}, \ldots, X_{n}$ are independent normal random variables with $X_{1}, X_{2}, \ldots, X_{m}$ are $N\left(\phi_{0}, \sigma^{2}\right)$ and $X_{m+1}, X_{m+2}, \ldots, X_{n}$ are $N\left(\phi_{1}, \sigma^{2}\right)$ where $\phi_{0}$ and $\phi_{1}$ are real unknown constants which represent the means of the variables $X_{i}$ before and after the change-point $m$ respectively. The model can be written as follows

$$
\left\{\begin{array}{l}
X_{i}=\phi_{0}+\epsilon_{i} \text { if } i=1,2, \ldots, m \\
X_{i}=\phi_{1}+\epsilon_{i} \text { if } i=m+1, \ldots, n
\end{array}\right.
$$

Cherifa Belkacem : cherifabelkacem@yahoo.fr

Hocine Fellag: hfellag@yahoo.com 
where the $\epsilon_{i}$ are normal random independent errors with mean zero and unknown constant variance $\sigma^{2}$ and $m$ is an integer which lies between 1 and $n-1, n$ being the size of the sample.

The problem of change-point has a long history. Since the pioneer paper of Page (1954), many authors have treated this topic. The author used the cumulative sums called CUSUM techniques to detect change in the distribution of sum of random variables.

Also, Chernoff and Zacks (1964) and Kander and Zachs (1966) proposed a Bayesian nonparametric test to detect a change in the mean of a sum of independent random variables. Ferreira (1975), Broemeling (1985); Broemeling and Tsurumi (1986) and Andrews, et al. (1996) treated the change point problem in regression models. Menzefricke (1981) studied the case of sequence of independent random variables where the posterior densities of the change point and the variance ratio in different situations.

More recently, Fearnhead (2006) presented how to perform direct simulation from the posterior distribution of a class of multiple change-point models where the number of change points is unknown. Rigaill et al. (2012) derived exact, explicit and tractable formulae for the posterior distribution of variables such as the number of change-points or their positions.

Also, Fotopoulos et al. (2010) derived exact computable expressions for the asymptotic distribution of the change-point maximum likelihood estimator when a change in the mean occurred at an unknown point of a sequence of time-ordered independent Gaussian random variables. In their paper, the authors interpreted the conditional distribution of this estimator as a Bayesian solution to the change-point problem.

In this paper, our aim is to study the impact of a single outlier on the estimation of the change-point $m$ of the model (1) when one observation is contaminated. The Bayesian approach is our main procedure.

Assume that there exists a position $k$ such that $k \in\{1, \ldots, n\}$ and

$$
\left\{\begin{array}{l}
Y_{k}=X_{k}+\Delta \\
Y_{i}=X_{i},
\end{array} \forall i \in\{1, \ldots, n\} \text { with } \quad i \neq k\right.
$$

where the constant $\Delta$ is the magnitude of the contamination which occurs at a specified time, say $k$. Since the outlier can occur before or after the change-point, we will consider two cases where we derive the posterior density of the change point when an outlier occurs.

\section{Contamination before change-point}

In this case, we have $k \in\{1, \ldots, m\}$. Then the model is written as follows :

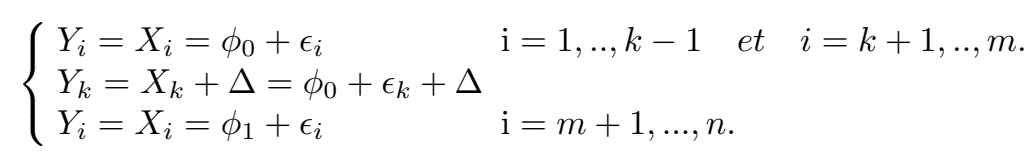

where: $\phi_{0} \neq \phi_{1}$, and $\epsilon_{i} \sim N\left(0, \sigma^{2}\right) ;(\sigma>0)$ avec $\phi_{0}, \phi_{1}$ et $\sigma$ are unknown parameters.

Assume that the priors of $\sigma^{2},\left(\phi_{0}, \phi_{1}\right)$ and the change-point $m$ are given by : 


$$
\begin{aligned}
& \pi_{0}\left(\sigma^{2}\right) \propto 1 / \sigma^{2} \\
& \pi_{0}\left(\phi_{0}, \phi_{1}\right) \propto \text { constant in } \boldsymbol{R}^{2} \\
& \pi_{0}(m) \propto 1 /(n-1) \text { for } m=1,2, \ldots, n-1 \\
& \sigma^{2},\left(\phi_{0}, \phi_{1}\right) \text { and } m \text { are independent. }
\end{aligned}
$$

In the following, we give, without proof, the following lemma.

Lemma 1. The likelihood function of the parameters $m, \sigma^{2}, \phi_{0}$ and $\phi_{1}$ is :

$$
\begin{aligned}
L\left(m, \sigma^{2}, \phi_{0}, \phi_{1} / Y\right) & \propto\left(\sigma^{2}\right)^{-n / 2} \exp \left\{-\frac{1}{2 \sigma^{2}}\left[\sum_{i=1}^{k-1}\left(Y_{i}-\phi_{0}\right)^{2}+\left(Y_{k}-\left(\phi_{0}+\Delta\right)\right)^{2}\right.\right. \\
& \left.\left.+\sum_{i=k+1}^{m}\left(Y_{i}-\phi_{0}\right)^{2}+\sum_{m+1}^{n}\left(Y_{i}-\phi_{1}\right)^{2}\right]\right\} .
\end{aligned}
$$

Lemma 2. The joint posterior density of $\phi_{0}, \phi_{1}, \sigma^{2}$ and $m$ is

$$
\pi_{1}\left(m, \sigma^{2}, \phi_{0}, \phi_{1}\right) \propto \text { prior } x \text { likelihood function }
$$

Then,

$$
\begin{aligned}
\pi_{1}\left(m, \sigma^{2}, \phi_{0}, \phi_{1}\right) & \propto\left(\sigma^{2}\right)^{-n / 2-1} \exp \left\{-\frac{1}{2 \sigma^{2}}\left[\sum_{i=1}^{k-1}\left(Y_{i}-\phi_{0}\right)^{2}+\left(Y_{k}-\left(\phi_{0}+\Delta\right)\right)^{2}\right.\right. \\
& \left.\left.+\sum_{i=k+1}^{m}\left(Y_{i}-\phi_{0}\right)^{2}+\sum_{m+1}^{n}\left(Y_{i}-\phi_{1}\right)^{2}\right]\right\} .
\end{aligned}
$$

\section{Proof}

The posterior joint density of the parameters $\left(m, \sigma^{2}, \phi_{0}, \phi_{1}\right)$ is derived using the following Bayes procedure :

$$
\pi_{1}\left(m, \sigma^{2}, \phi_{0}, \phi_{1}\right) \propto L\left(m, \sigma^{2}, \phi_{0}, \phi_{1} / Y\right) . \pi_{0}\left(m, \sigma^{2}, \phi_{0}, \phi_{1}\right)
$$

Since $\left(m, \sigma^{2}, \phi_{0}, \phi_{1}\right)$ are independent, we can write:

$$
\pi_{0}\left(m, \sigma^{2}, \phi_{0}, \phi_{1}\right) \propto \frac{1}{\sigma^{2}}
$$

The likelihood function of the parameters $m, \sigma^{2}, \phi_{0}$ and $\phi_{1}$ is given in lemma 2.1. Then,

$$
\begin{aligned}
\pi_{1}\left(m, \sigma^{2}, \phi_{0}, \phi_{1}\right) & \propto\left(\sigma^{2}\right)^{-n / 2-1} \exp \left\{-\frac{1}{2 \sigma^{2}}\left[\sum_{i=1}^{k-1}\left(Y_{i}-\phi_{0}\right)^{2}+\left(Y_{k}-\left(\phi_{0}+\Delta\right)\right)^{2}\right.\right. \\
& \left.\left.+\sum_{i=k+1}^{m}\left(Y_{i}-\phi_{0}\right)^{2}+\sum_{m+1}^{n}\left(Y_{i}-\phi_{1}\right)^{2}\right]\right\}
\end{aligned}
$$

Journal home page: www.jafristat.net 
Proposition 1. Under the conditions (3) given above, the posterior density of the changepoint $m$ is as follows :

$$
\pi_{1}(m) \propto[m(n-m)]^{-\frac{1}{2}} \cdot Q_{2}(\Delta)^{-\frac{n-2}{2}}
$$

where

$Q_{2}(\Delta)=-\frac{\left(\sum_{i=1}^{m} Y_{i}-\Delta\right)^{2}}{m}+\frac{\left(\sum_{i=1}^{k-1} Y_{i}\right)^{2}}{k-1}+\frac{\left(\sum_{i=k+1}^{m} Y_{i}\right)^{2}}{m-k}+\left(Y_{k}-\Delta\right)^{2}+S_{1}^{k-1}+S_{k+1}^{m}+S_{m+1}^{n}$

with $\quad S_{j}^{k}=\sum_{i=j}^{k}\left(Y_{i}-\bar{Y}_{j}^{k}\right)^{2}$ and $\quad \bar{Y}_{j}^{k}=\frac{1}{k-j+1} \sum_{i=j}^{k} Y_{i}$, for $k=1, \ldots, n$.

\section{Proof}

$$
\pi_{1}(m) \propto \int_{0}^{+\infty} \int_{R} \int_{R} \pi_{1}\left(m, \sigma^{2}, \phi_{0}, \phi_{1}\right) d \phi_{0} d \phi_{1} d \sigma^{2}
$$

The integral with respect $\sigma^{2}$ allows us to derive the posterior density of $m, \phi_{0}$ and $\phi_{1}$.

$$
\pi_{1}\left(m, \phi_{0}, \phi_{1}\right)=\int_{0}^{\infty} \pi_{1}\left(m, \sigma^{2}, \phi_{0}, \phi_{1}\right) d \sigma^{2}
$$

If we assume that $\sigma^{2}=\frac{1}{r}$, we obtain $d \sigma^{2}=-1 / r^{2} d r$ and then,

$$
\begin{aligned}
\pi_{1}\left(m, \phi_{0}, \phi_{1}\right) & \propto \int_{0}^{+\infty} r^{n / 2-1} \cdot \exp \left\{-\frac{r}{2}\left[\sum_{i=1}^{k-1}\left(Y_{i}-\phi_{0}\right)^{2}+\left(Y_{k}-\left(\Delta+\phi_{0}\right)\right)^{2}\right.\right. \\
& \left.\left.+\sum_{i=k+1}^{m}\left(Y_{i}-\phi_{0}\right)^{2}+\sum_{i=m+1}^{n}\left(Y_{i}-\phi_{1}\right)^{2}\right]\right\} d r
\end{aligned}
$$

Using the properties of the gamma distribution, $\left(\int_{0}^{\infty}\left(b^{a} / \Gamma(a)\right) r^{a-1} e^{-b r} d r=1\right)$, we obtain

$$
\pi_{1}\left(m, \phi_{0}, \phi_{1}\right) \propto\left[\sum_{i=1}^{k-1}\left(Y_{i}-\phi_{0}\right)^{2}+\left(Y_{k}-\left(\Delta+\phi_{0}\right)\right)^{2}+\sum_{i=k+1}^{m}\left(Y_{i}-\phi_{0}\right)^{2}+\sum_{i=m+1}^{n}\left(Y_{i}-\phi_{1}\right)^{2}\right]^{-\frac{n}{2}}
$$

To obtain $\pi_{1}\left(m, \phi_{0}\right)$, we have to perform the integral with respect to $\phi_{1}$,

$$
\pi_{1}\left(m, \phi_{0}\right)=\int_{R} \pi_{1}\left(m, \phi_{0}, \phi_{1}\right) d \phi_{1}
$$

Assume that,

$$
I=\sum_{i=1}^{k-1}\left(Y_{i}-\phi_{0}\right)^{2}+\left(Y_{k}-\left(\Delta+\phi_{0}\right)\right)^{2}+\sum_{i=k+1}^{m}\left(Y_{i}-\phi_{0}\right)^{2}+\sum_{i=m+1}^{n}\left(Y_{i}-\phi_{1}\right)^{2}
$$


The expression can be rewritten as :

$$
\begin{aligned}
I & =\sum_{i=1}^{k-1}\left(Y_{i}-\bar{Y}_{1}^{k-1}+\bar{Y}_{1}^{k-1}-\phi_{0}\right)^{2}+\left(Y_{k}-\Delta-\phi_{0}\right)^{2} \\
& +\sum_{i=k+1}^{m}\left(Y_{i}-\bar{Y}_{k+1}^{m}+\bar{Y}_{k+1}^{m}-\phi_{0}\right)^{2}+\sum_{i=m+1}^{n}\left(Y_{i}-\bar{Y}_{m+1}^{n}+\bar{Y}_{m+1}^{n}-\phi_{1}\right)^{2}
\end{aligned}
$$

with $\bar{Y}_{j}^{k}=\frac{1}{k-j+1} \sum_{i=j}^{k} Y_{i}$. We have:

$$
\begin{aligned}
2 \sum_{i}^{k-1}\left[\left(Y_{i}-\bar{Y}_{1}^{k-1}\right)\left(\bar{Y}_{1}^{k-1}-\phi_{0}\right)\right] & =2 \sum_{k+1}^{m}\left[\left(Y_{i}-\bar{Y}_{k+1}^{m}\right)\left(\bar{Y}_{k+1}^{m}-\phi_{0}\right)\right] \\
& =2 \sum_{m+1}^{n}\left[\left(Y_{i}-\bar{Y}_{m+1}^{n}\right)\left(\bar{Y}_{m+1}^{n}-\phi_{1}\right)\right]=0
\end{aligned}
$$

and

$$
\begin{aligned}
I & =(k-1)\left(\bar{Y}_{1}^{k-1}-\phi_{0}\right)^{2}+(m-k)\left(\bar{Y}_{k+1}^{m}-\phi_{0}\right)^{2}+(n-m)\left(\bar{Y}_{m+1}^{n}-\phi_{1}\right)^{2} \\
& +S_{1}^{k-1}+S_{k+1}^{m}+S_{m+1}^{n}+\left(Y_{k}-\phi_{0}-\Delta\right)^{2} \\
& =Q_{1}(\Delta)+S_{m+1}^{n}+(n-m)\left(\bar{Y}_{m+1}^{n}-\phi_{1}\right)^{2}
\end{aligned}
$$

where $S_{j}^{k}=\sum_{i=j}^{k}\left(Y_{i}-\bar{Y}_{j}^{k}\right)$ and

$$
\begin{aligned}
Q_{1}(\Delta) & =(k-1)\left(\bar{Y}_{1}^{k-1}-\phi_{0}\right)^{2}+(m-k)\left(\bar{Y}_{k+1}^{m}-\phi_{0}\right)^{2} \\
& +\left(Y_{k}-\phi_{0}-\Delta\right)^{2}+S_{1}^{k-1}+S_{k+1}^{m}
\end{aligned}
$$

Thus,

$$
\begin{aligned}
\pi_{1}\left(m, \phi_{0}\right) & \propto \int_{R}\left[Q_{1}(\Delta)+S_{m+1}^{n}+(n-m)\left(\bar{Y}_{m+1}^{n}-\phi_{1}\right)^{2}\right]^{-\frac{n}{2}} d \phi_{1} \\
& =\left(Q_{1}(\Delta)+S_{m+1}^{n}\right)^{-\frac{n}{2}} \int_{R}\left[1+\left(\frac{\bar{Y}_{m+1}^{n}-\phi_{1}}{\sqrt{\left(Q_{1}(\Delta)+S_{m+1}^{n}\right) /(n-m)}}\right)^{2}\right]^{-\frac{n}{2}} d \phi_{1} .
\end{aligned}
$$

Assume that

$$
\frac{z}{\sqrt{n-1}}=\frac{\bar{Y}_{m+1}^{n}-\phi_{1}}{\sqrt{\left(Q_{1}(\Delta)+S_{m+1}^{n}\right) /(n-m)}}
$$


Using the properties of $t$-distribution with $n$ degrees of freedom, we obtain,

$$
\begin{aligned}
\pi_{1}\left(m, \phi_{0}\right) & \propto\left(Q_{1}(\Delta)+S_{m+1}^{n}\right)^{-\frac{n-1}{2}}(n-1)^{-\frac{1}{2}}(n-m)^{-\frac{1}{2}} \int_{R}\left(1+\frac{z^{2}}{n-1}\right)^{-\frac{n}{2}} d z \\
& \propto\left(Q_{1}(\Delta)+S_{m+1}^{n}\right)^{-\frac{n-1}{2}} \cdot[(n-m)(n-1)]^{-\frac{1}{2}}(n-1)^{\frac{1}{2}}
\end{aligned}
$$

which leads to,

$$
\pi_{1}\left(m, \phi_{0}\right) \propto\left(Q_{1}(\Delta)+S_{m+1}^{n}\right)^{-\frac{n-1}{2}}(n-m)^{-\frac{1}{2}} .
$$

An other integration with respect to $\phi_{0}$ gives the posterior density of change point $m$.

$$
\pi_{1}(m) \propto \int_{R}\left(Q_{1}(\Delta)+S_{m+1}^{n}\right)^{-\frac{n-1}{2}}(n-m)^{-\frac{1}{2}} d \phi_{0} .
$$

Then, we have

$$
\begin{aligned}
Q_{1}(\Delta)+S_{m+1}^{n} & =(k-1)\left(\bar{Y}_{1}^{k-1}-\phi_{0}\right)^{2}+(m-k)\left(\bar{Y}_{k+1}^{m}-\phi_{0}\right)^{2} \\
& +\left(Y_{k}-\phi_{0}-\Delta\right)^{2}+S_{1}^{k-1}+S_{k+1}^{m}+S_{m+1}^{n} \\
& =m \phi_{0}^{2}-2 \phi_{0}\left(\sum_{i=1}^{m} Y_{i}-\Delta\right)+\frac{\left(\sum_{i=1}^{k-1} Y_{i}\right)^{2}}{k-1}+\frac{\left(\sum_{i=k+1}^{m} Y_{i}\right)^{2}}{m-k} \\
& +\left(Y_{k}-\Delta\right)^{2}+S_{1}^{k-1}+S_{k+1}^{m}+S_{m+1}^{n} \\
& =m\left[\phi_{0}-\frac{\sum_{i=1}^{m} Y_{i}-\Delta}{m}\right]^{2}-\frac{\left(\sum_{i=1}^{m} Y_{i}-\Delta\right)^{2}}{m}+\frac{\left(\sum_{i=1}^{k-1} Y_{i}\right)^{2}}{k-1}+\frac{\left(\sum_{i=k+1}^{m} Y_{i}\right)^{2}}{m-k} \\
& +\left(Y_{k}-\Delta\right)^{2}+S_{1}^{k-1}+S_{k+1}^{m}+S_{m+1}^{n}
\end{aligned}
$$

and,

$$
Q_{1}(\Delta)+S_{m+1}^{n}=m\left[\phi_{0}-\frac{\sum_{i=1}^{m} Y_{i}-\Delta}{m}\right]^{2}+Q_{2}(\Delta)
$$

where

$Q_{2}(\Delta)=-\frac{\left(\sum_{i=1}^{m} Y_{i}-\Delta\right)^{2}}{m}+\frac{\left(\sum_{i=1}^{k-1} Y_{i}\right)^{2}}{k-1}+\frac{\left(\sum_{i=k+1}^{m} Y_{i}\right)^{2}}{m-k}+\left(Y_{k}-\Delta\right)^{2}+S_{1}^{k-1}+S_{k+1}^{m}+S_{m+1}^{n}$

Thus,

$$
\pi_{1}(m) \propto \int_{R}(n-m)^{-\frac{1}{2}}\left[m\left(\phi_{0}-\frac{\sum_{i=1}^{m} Y_{i}-\Delta}{m}\right)^{2}+Q_{2}(\Delta)\right]^{-\frac{n-1}{2}} d \phi_{0}
$$

We can write, 


$$
\pi_{1}(m) \propto(n-m)^{-\frac{1}{2}} \cdot Q_{2}(\Delta)^{-\frac{n-1}{2}} \int_{R}\left[1+\left(\frac{\phi_{0}-\frac{\sum_{i=1}^{m} Y_{i}-\Delta}{m}}{\sqrt{Q_{2}(\Delta) / m}}\right)^{2}\right]^{-\frac{n-1}{2}} d \phi_{0}
$$

Assume that

$$
\frac{z}{\sqrt{n-2}}=\left(\phi_{0}-\frac{\sum_{i=1}^{m} Y_{i}-\Delta}{m}\right) / \sqrt{Q_{2}(\Delta) / m}
$$

Finally,

$$
\pi_{1}(m) \propto(n-m)^{-\frac{1}{2}} \cdot Q_{2}(\Delta)^{-\frac{n-1}{2}} \cdot \frac{\sqrt{Q_{2}(\Delta) / m}}{\sqrt{n-2}} \int_{R}\left(1+\frac{z^{2}}{n-2}\right)^{-\frac{n-1}{2}} d z
$$

Since $\int_{R}\left(1+\frac{z^{2}}{n-2}\right)^{-\frac{n-1}{2}} d z \propto(n-2)^{1 / 2}(t$-distribution with $n-1$ degrees of freedom), the proof of the proposition is completed.

\section{Contamination after change-point}

Now, the model is written as follows

$$
\begin{cases}Y_{i}=X_{i}=\phi_{0}+\epsilon_{i} & \mathrm{i}=1, \ldots, m . \\ Y_{i}=X_{i}=\phi_{1}+\epsilon_{i} & \mathrm{i}=m+1, \ldots, k-1 \quad \text { and } \quad i=k+1, \ldots, n . \\ Y_{k}=X_{k}+\Delta=\phi_{1}+\epsilon_{k}+\Delta & \end{cases}
$$

where $\phi_{0} \neq \phi_{1}, \epsilon_{i} \sim N\left(0, \sigma^{2}\right)$ and $\sigma>0$ with $\phi_{0}, \phi_{1}$ and $\sigma$ unknown parameters.

In this case, we can use the same methodology than the section 2 with the following difference: here, we have

$$
\pi_{1}\left(m, \sigma^{2}, \phi_{0}, \phi_{1}\right) \propto L\left(m, \sigma^{2}, \phi_{0}, \phi_{1} / Y\right) \cdot \pi_{0}\left(m, \sigma^{2}, \phi_{0}, \phi_{1}\right)
$$

and

Since

$$
\pi_{0}\left(m, \sigma^{2}, \phi_{0}, \phi_{1}\right) \propto \frac{1}{\sigma^{2}}
$$

$Y_{i}$ is distributed according to $N\left(\phi_{0}, \sigma^{2}\right)$ for $i=1, \ldots, m$.

$Y_{i}$ is distributed according to $N\left(\phi_{1}, \sigma^{2}\right)$ for $i=m+1, . ., k-1$ or $i=k+1, \ldots, n$.

$Y_{k}$ is distributed according to $N\left(\phi_{1}+\Delta, \sigma^{2}\right)$

$$
\begin{aligned}
L\left(m, \sigma^{2}, \phi_{0}, \phi_{1} / Y\right) & \propto\left(\sigma^{2}\right)^{-n / 2} \exp \left\{-\frac{1}{2 \sigma^{2}}\left[\sum_{i=1}^{m}\left(Y_{i}-\phi_{0}\right)^{2}+\left(Y_{k}-\left(\phi_{1}+\Delta\right)\right)^{2}\right.\right. \\
& \left.\left.+\sum_{i=m+1}^{k-1}\left(Y_{i}-\phi_{1}\right)^{2}+\sum_{k+1}^{n}\left(Y_{i}-\phi_{1}\right)^{2}\right]\right\}
\end{aligned}
$$

Journal home page: www.jafristat.net 
and

$$
\begin{aligned}
\pi_{1}\left(m, \sigma^{2}, \phi_{0}, \phi_{1}\right) & \propto\left(\sigma^{2}\right)^{n / 2-1} \exp \left\{-\frac{1}{2 \sigma^{2}}\left[\sum_{i=1}^{m+1}\left(Y_{i}-\phi_{0}\right)^{2}+\left(Y_{k}-\left(\phi_{1}+\Delta\right)\right)^{2}\right.\right. \\
& \left.\left.+\sum_{i=m+1}^{k-1}\left(Y_{i}-\phi_{0}\right)^{2}+\sum_{k+1}^{n}\left(Y_{i}-\phi_{1}\right)^{2}\right]\right\} .
\end{aligned}
$$

Then, we derive the expression of the posterior density of the change-point $m$ as follows

$$
\pi_{1}(m) \propto[m(n-m)]^{-\frac{1}{2}} \cdot\left[Q_{2}(\Delta)\right]^{-\frac{n-2}{2}}
$$

with

$Q_{2}(\Delta)=-\frac{\left(\sum_{i=m+1}^{n} Y_{i}-\Delta\right)^{2}}{n-m}+\frac{\left(\sum_{i=m+1}^{k-1} Y_{i}\right)^{2}}{k-m-1}+\frac{\left(\sum_{i=k+1}^{n} Y_{i}\right)^{2}}{n-k}+\left(Y_{k}-\Delta\right)^{2}+S_{1}^{m}+S_{m+1}^{k-1}+S_{k+1}^{n}$

\section{Numerical study}

To illustrate the impact of outliers on change-point problems, we present in this section some results using simulation procedures. We simulated 100 samples from the contaminated model (2) where $\phi_{0}=0, \phi_{1}=1$ and $\sigma^{2}=0.5$ for different values of the magnitude $\Delta$. The sample sizes $N=25,50,100$ are considered. In our simulations, we noted that position of the outlier and of the change-point do not play any role.

In the Fig.1, we assume that $N=25, k=5$ and $m=15$. One can remark that, in the case of sample size, the method is sensitive to slight deviations of $\Delta$ (see Fig. 1 (c) ). Also, the method is inefficient for $\Delta \geq 3$.
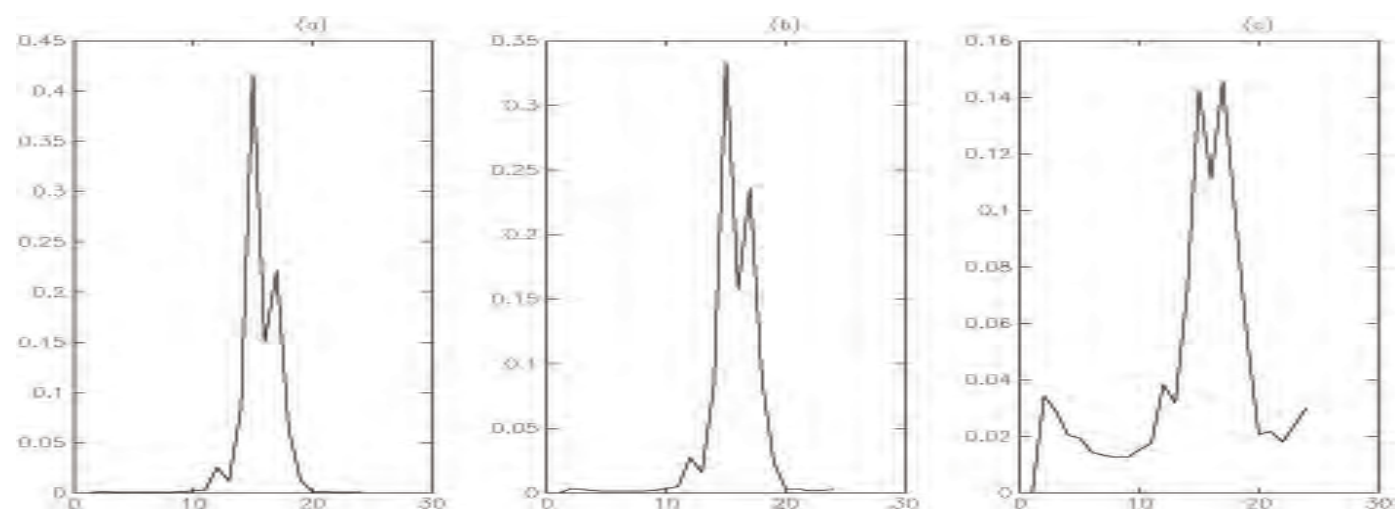

Fig. 1. Variation of the change-point estimation with $\Delta$ for $N=25$ and $m=15$

$$
(a): \Delta=0,(b): \Delta=1,(c): \Delta=3
$$


In the Fig.2, we present the variation of the change-point estimation with the magnitude $\Delta$ when $N=50, m=20$ and $k=10$. We remark that the performance of the method is not satisfactory for $\Delta \geq 5$.
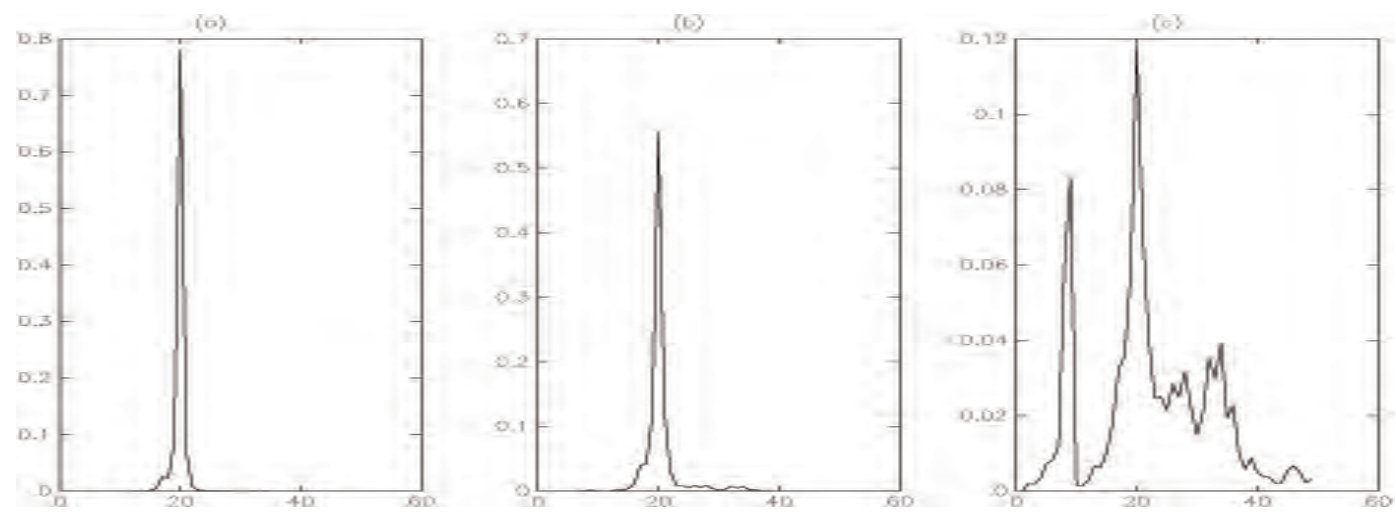

Fig. 2. Variation of the change-point estimation with $\Delta$ for $N=50$ and $m=20$

$$
(a): \Delta=0,(b): \Delta=3,(c): \Delta=5
$$

Finally, the Fig. 3 presents the case of $N=100, m=30$ and $k=10$. One can notice that the method is insensitive until the value of $\Delta=14$ only. Then, for slight deviations of the magnitude, the method is stable. Also, one can say that the Bayesian procedure used in this paper shows that the estimation is insensitive for high sample sizes but is rather useless in small sample case.
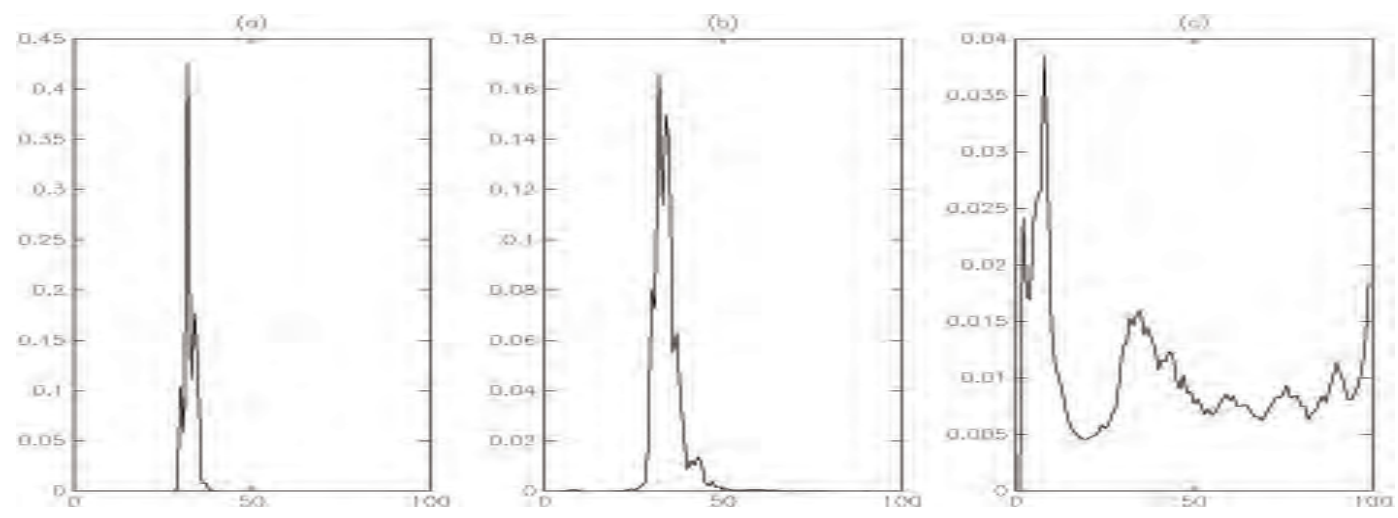

Fig. 3. Variation of the change-point estimation with $\Delta$ for $N=100$ and $m=15$.

$(a): \Delta=0,(b): \Delta=11,(c): \Delta=14$ 


\section{Concluding remarks}

In the case of small samples, an outlier has a significant impact on the Bayesian procedure of estimation of the change-point. In this case, the estimated change-point depends on the magnitude of the outlier. However, if the sample size is high, this influence is not important and makes the procedure stable in the presence of a contaminant. For further investigations, it would be interesting to reconsider this work assuming that the magnitude is a random variable.

Acknowledgments. We thank the referee for his helpful comments.

\section{References}

Andrews, D.W.K., Lee, I. and Ploberger, W., 1996. Optimal changepoint tests for normal linear regression, J. Econometrics, 70(1), 9-38.

Broemeling, L.D. and Tsurumi, H., 1986. Econometrics and structural changes. Marcel Dekker Inc Eds, New York.

Broemeling, L.D., 1985. The Bayesian analysis of linear model. Marcel Dekker Inc Eds, New York.

Chernoff, H. and Zacks, S., 1964. Estimating the current mean of a normal distribution which is subjected to changes in time, Annal of Mathematical Statistics. 35, 999-1018.

Fearnhead, P., 2006. Exact and efficient Bayesian inference for multiple changepoint problems. Statistics and computing. 16, 203-213.

Ferreira, P.E. 1975. Bayesian analysis of a switching regression model : known number of regimes, Amer. Journ. Stat.Association. 70, 370-374.

Fotopoulos, S.B, Jandhyala,V.K., and Khapalova, E., 2010. Exact asymptotic distribution of change-point mle for change in the mean of Gaussian sequences.Annals of Applied Statistics, 4(2), 1081-1104.

Kander, Z. and Zachs, S., 1966. Test procedures for possible changes in parameters of statistical distribution occuring at unknown time points, Ann. Math. Stat., 37, 1196-1210.

Menzefricke, U., 1981. A Bayesian Analysis of a change in the precision of a sequence of independent normal random variable at an unknown time point, Applied Statistics, 30, 141-146.

Page, E.S., 1954. Continuous inspection shemes, Biometrika, 41, 100-115.

Rigaill, G., Lebarbier, E. and Robin, S., 2012. Exact posterior distributions and model selection criteria for multiple change-point detection problems. Stat. Comput. 22(4), 917929. 